\title{
Actitudes de las enfermeras frente a la muerte de los pacientes en una unidad de cuidados intensivos
}

\author{
Attitudes of Nurses towards the Death of \\ Patients in an Intensive Care Unit
}

\section{Atitudes das enfermeiras frente à morte dos pacientes em uma unidade de cuidados intensivos}

Diana Isabel Cáceres Rivera, $\mathrm{PhD}^{1 *}$

Luisa Yaneth Cristancho Zambrano, $\mathrm{RN}^{2}$

Luis Alberto López Romero, $\mathrm{MSc}^{3}$

Recibido: $1^{\circ}$ de abril de 2019 - Aceptado: 11 de junio de 2019

Doi: http://dx.doi.org/10.12804/revistas.urosario.edu.co/revsalud/a.8368

Para citar este artículo: Cáceres Rivera DI, Cristancho LF, López Romero LA. Actitudes de las enfermeras frente a la muerte de los pacientes en una unidad de cuidados intensivos. Rev Cienc Salud. 2019;17(3):98-110. Doi: http://dx.doi.org/10.12804/revistas.urosario. edu.co/revsalud/a.8368

\section{Resumen}

Introducción: en las unidades de cuidado intensivo (ucI) es frecuente el contacto con la muerte a la que se expone el profesional de enfermería. Este tema ha sido poco estudiado en nuestro país. El objetivo de este estudio fue describir las actitudes hacia la muerte en un grupo de enfermeras que laboraban en una uci de alta complejidad. Materiales y métodos: estudio descriptivo en el que se incluyeron 17 profesionales de enfermería que trabajan en una unidad de cuidados intensivos de alta complejidad. Se aplicó el perfil revisado de actitudes hacia la muerte. Las variables continuas fueron reportadas como promedio acompañadas de desviación estándar, dado que tenían una distribución normal según la prueba de Shapiro-Wilk y demás pruebas gráficas. Las variables categóricas fueron expresadas como frecuencias absolutas o relativas. Resultados: el promedio general de la escala de actitud hacia la muerte fue 133,53 $\pm 21,50$. La actitud con mayor promedio fue la de aceptación de acercamiento con 48,17 \pm 12,64, seguida por la actitud de aceptación neutral con 28,94 $\pm 4,62$ y miedo a la muerte con $23,0 \pm 7,42$. Existe una correlación moderada y directa entre el promedio

1 Grupo Gifosabi, Facultad de Enfermería, Universidad Cooperativa de Colombia, Bucaramanga (Santander, Colombia).

Autor de correspondencia: dianai.caceres@ucc.edu.co

2 Unidad de Cuidados Intensivos, Fundación Cardiovascular de Colombia-Hospital Internacional, Piedecuesta (Santander, Colombia).

3 Fundación Cardiovascular de Colombia. Grupo de Investigación y Desarrollo de Conocimiento en Enfermería FCV (GIDCEN-FCV). Centro de investigaciones, Floridablanca (Santander, Colombia). 
general de la escala de actitudes hacia la muerte y los años de trabajo en el servicio, rho = 0,59, $p=0,016$. Conclusiones: dentro de las actitudes hacia la muerte de los participantes del presente estudio, la que más predominó fue la de aceptación de acercamiento. Adicionalmente, se pudo establecer que existe una correlación moderada y directa entre el promedio general de la escala de actitudes hacia la muerte y los años de trabajo en ucI.

Palabras clave: enfermeros, actitud frente a la muerte, unidad de cuidados intensivos.

\section{Abstract}

Introduction: In intensive care units (IcU), contact with death is frequent for nurses who work there. In our country, this issue has been little studied. The objective of this study was to describe attitudes towards death in a group of nurses who work in a highly complex Icu. Materials and methods: This descriptive study included 17 nursing professionals working in a highly complex Icu. The revised profile of attitudes toward death was applied. Continuous variables were reported as average accompanied by standard deviation, given that they had a normal distribution according to the Shapiro-Wilk test and other graphic ones. Categorical variables were expressed as absolute or relative frequencies. Results: The overall average of the attitude towards death scale was $133.53 \pm 21.50$. The attitude with the highest average was the acceptance approach with $48.17 \pm 12.64$, followed by neutral acceptance with $28.94 \pm$ 4.62, and fear of death with $23.0 \pm 7.42$. There is a moderate and direct correlation between the general average of the scale of attitudes toward death and years of work in the service, rho $=0.59, p=0.016$. Conclusions: Among the participants' attitudes towards death, the one that prevailed most was acceptance approach. Additionally, it was established that there is a moderate and direct correlation between the general average of the scale of attitudes towards death and the years of work in the Icu.

Keywords: Nurses, attitude to death, intensive care unit.

\section{Resumo}

Introdução: nas unidades de cuidado intensivo (UcI) é frequente o contacto com a morte à que se expõe o profissional de enfermagem. Este tema tem sido pouco estudado em nosso país. O objetivo deste estudo foi descrever as atitudes à morte em um grupo de enfermeiras que trabalha em uma uci de alta complexidade. Materiais e métodos: estudo descritivo no que se incluíram 17 profissionais de enfermagem que trabalham em uma unidade de cuidados intensivos de alta complexidade. Se aplicou o perfil revisado de atitudes à morte. As variáveis contínuas foram reportadas como média acompanhadas de desvio-padrão, dado que tinham uma distribuição normal segundo a prova de Shapiro-Wilk e demais provas gráficas. As variáveis categóricas foram expressadas como frequências absolutas ou relativas. Resultados: a média geral da escala de atitude à morte foi 133,53 $\pm 21,50$. A atitude com maior média foi a de aceitação aproximação com $48,17 \pm 12,64$, seguida pela atitude de aceitação neutral com $28,94 \pm 4,62$ e medo à morte com 23,0 \pm 7,42. Existe uma correlação moderada e direta entre a média geral da escala de atitudes à morte e os anos de trabalho no serviço, rho = 0,59, p = 0,016. Conclusões: dentro das atitudes à norte dos participantes do presente estudo, a que mais predominou foi a de abordagem de aceitação. Adicionalmente, se conseguiu estabelecer que existe uma correlação moderada e direta entre a média geral da escala de atitudes à morte e os anos de trabalho em uci.

Palavras-chave: enfermeiros, atitude frente à morte, unidade de cuidados intensivos. 


\section{Introducción}

T as unidades de cuidado intensivo son servicios cuyo objetivo es proveer la atención de ـmás alta calidad a los pacientes que se encuentran en un estado de salud críticamente comprometido. En ese contexto, y dadas sus condiciones clínicas, es preponderante una relación enfermera-paciente racional en términos de calidad y satisfacción de la demanda de cuidados (1).

Al ser unidades de pacientes en estado crítico, existe una mortalidad elevada en las unidades de cuidado intensivo (ucI) en comparación con otras unidades o servicios. Se ha descrito, por ejemplo, en Colombia, que existe una alta tasa de mortalidad de estos pacientes, secundaria a la prevalencia de diagnósticos cardiovasculares, los cuales generalmente presentan un mal pronóstico. Esto refleja la cotidianidad de contacto con la muerte a la que se ven enfrentados diariamente los profesionales de enfermería que trabajan en dichas unidades (2). Adicionalmente, este escenario de desempeño profesional se caracteriza por otros eventos asociados a la muerte, como lo son la enfermedad terminal, la agonía, el dolor y el sufrimiento, haciendo de las uci ambientes laborales con altos niveles de estrés, ansiedad, entre otros (3).

Estas características de las uci generan ciertas repercusiones para el profesional de enfermería, tal y como lo describió Zambrano en su estudio sobre estresores en las unidades de cuidado intensivo, en donde describe el contacto con el dolor, la toma de decisiones que pueden comprometer la vida del paciente y la muerte como principales estresores, lo cual está fundamentado en la responsabilidad sobre la vida, la salud y el cuidado de otras personas (4).

En razón a lo anteriormente expuesto, algunos autores, como Díaz y colaboradores, refieren que los profesionales de enfermería que laboran en unidades de cuidado intensivo adoptan ciertas actitudes hacia la muerte que pueden ser causadas por un agotamiento emocional, por la ansiedad y por el estrés (5). Estas actitudes que el profesional de enfermería toma frente a la muerte dependen de varios factores. Alonso, en 2014, define la muerte como un suceso que ocasiona todo tipo de comportamientos, actitudes y emociones, como el miedo y la ansiedad en la mayor parte de las ocasiones (6).

La unidad de cuidado intensivo se convierte entonces en un espacio que permite observar la capacidad de afrontamiento del profesional de enfermería, la cual se ve reflejada en la actitud hacia la muerte, siendo importante conocer el impacto que estas situaciones tienen en la enfermera y su sujeto de cuidado. Para tal fin existen instrumentos validados que miden efectos como la ansiedad o determinan actitudes de las enfermeras, por ejemplo, el Perfil de Actitudes hacia la Muerte (PAM-R), que se encuentra validado para Colombia, ofreciendo una herramienta ideal para la descripción de las actitudes $(7,8)$.

Con el fin de tener un primer acercamiento a este tema, el objetivo del presente artículo fue describir las actitudes hacia la muerte en un grupo de enfermeras que labora en una unidad de cuidados intensivos de un hospital de alta complejidad. 


\section{Materiales y métodos}

C e llevó a cabo un estudio descriptivo en el que se incluyó la totalidad de los profesionales de enfermería (n=17) de la unidad de cuidados intensivos de adultos de una institución de alta complejidad en la ciudad de Bucaramanga (Colombia). Se tuvieron en cuenta aquellos profesionales que llevaran más de un año de antigüedad en la uci y más de dos años de ejercicio laboral como profesionales. Como criterios de exclusión se estableció no incluir personas que estuvieran en un período de duelo por pérdida de un familiar o persona cercana en los últimos seis meses, o a quienes en el momento de la entrevista conocieran directamente a uno de los pacientes internados en la uci. Esta situación no se presentó en ninguno de los participantes. Todos los participantes del estudio firmaron un consentimiento informado; así mismo, el estudio fue aprobado por el comité de ética de la institución involucrada. Igualmente, se cumplió con la normatividad nacional e internacional para la investigación en seres humanos.

Para la valoración de las actitudes hacia la muerte, se aplicó el PAM-R, elaborado por Gesser, Wong y Reker en el año 1988, validado previamente en población colombiana (9). Esta escala contiene afirmaciones relacionadas con cinco tipos de actitudes y tienen escalamiento tipo Likert, con siete opciones de respuesta que van desde totalmente en desacuerdo a totalmente de acuerdo $(1,7)$. Para cada tipo de actitud se puede calcular una puntuación media de la escala dividiendo la puntuación total por el número de ítems que la forman. Este instrumento consta de cinco dimensiones evaluativas (actitudes), que junto a sus ítems se hallan ordenadas así:

Componente 1: aceptación de acercamiento $(4,8,13,15,16,22,25,27,28,31)$

Componente 2: miedo a la muerte $(1,2,7,18,20,21,32)$

Componente 3: evitación de la muerte $(3,10,12,19,26)$

Componente 4: aceptación de escape $(5,9,11,23,29)$

Componente 5: aceptación neutral $(6,14,17,24,30)$

La aceptación de acercamiento se relaciona con creencias religiosas, en donde se tiene una mayor confianza y está presente la creencia de una vida feliz después de la muerte.

El miedo a la muerte se refiere específicamente a la respuesta consciente que tiene el ser humano ante la muerte, en la cual las variables mediadoras se relacionan con el temor a la pérdida de sí mismo, a lo desconocido, al más allá de la muerte, al dolor y al sufrimiento, al bienestar de los miembros supervivientes de la familia. El miedo surge como resultado de la falta de significado de la propia vida y de la propia muerte.

La evitación ante la muerte se asocia con la actitud de rechazo ante el tema de la muerte y toda situación, diálogo o lugar que se vincule con esta.

La aceptación de escape se presenta en las personas que carecen de habilidades de afrontamiento eficaz para enfrentar el dolor o los problemas de la existencia, por lo tanto, la muerte se puede convertir en una alternativa y puede ofrecer el único escape ante las emociones negativas. 
La aceptación neutral es definida como la ambivalencia presente en la persona frente al tema de la muerte, expresada en un temor normal a ella, pero también en su aceptación ante su posible llegada, en donde la reconoce como un hecho innegable en la vida y se intenta aprovechar al máximo la existencia que se tiene.

La escala PAM-R en sus subescalas tiene coeficientes de confiabilidad adecuados: miedo (= $0,86)$, evitación $(=0,88)$, neutral $(=0,65)$, acercamiento $(=0,97)$, escape $(=0,84)(9)$.

El presente análisis se realizó conforme con la normatividad vigente para estudios en humanos, por lo cual contó con la respectiva aprobación de los comités de ética de las instituciones involucradas. De igual modo, luego de recibir una explicación completa sobre la investigación, se entregó y obtuvo el consentimiento informado de quienes de manera voluntaria accedieron a participar, en donde se describieron de forma detallada las características del estudio (10,11).

Posteriormente, se explicó el instrumento a los participantes, quienes autodiligenciaron los formatos de manera escrita y anónima. Durante este proceso los investigadores estuvieron atentos a inquietudes sobre el diligenciamiento de este. La información fue digitada en una base de datos de Excel previamente diseñada y codificada por los investigadores; dicha base de datos fue importada al programa estadístico STATA, versión 14.0, en donde fueron llevados a cabo todos los análisis estadísticos. Fue efectuado un análisis descriptivo, las variables cualitativas fueron expresadas como frecuencias absolutas o relativas, mientras que las variables continuas fueron reportadas como promedio acompañadas de desviación estándar, dado que tenían una distribución normal según la prueba de Shapiro-Wilk y sktest (tabla 1). Adicionalmente, se hizo un análisis de correlación de Spearman entre la percepción general de la escala PAM-R y los años de trabajo en la unidad de los participantes.

Tabla 1. Parámetros y probabilidades de las pruebas de Shapiro-Wilk y sktest

\begin{tabular}{lcccc}
\hline \multicolumn{1}{c}{ Variable } & \multicolumn{2}{c}{ Shapiro-Wilk } & \multicolumn{2}{c}{ Sktest } \\
\cline { 2 - 5 } & $\mathbf{Z}$ & Prob $>\mathbf{z}$ & $\mathbf{c h i}^{\mathbf{2}} \mathbf{( 2 )}$ & Prob $>\mathbf{c h i}^{\mathbf{2}}$ \\
\hline Aceptación de acercamiento & 1,415 & 0,079 & 8,45 & 0,0146 \\
Evitación de la muerte & 0,753 & 0,226 & 2,19 & 0,334 \\
Aceptación de escape & 1,332 & 0,091 & 1,78 & 0,410 \\
Miedo a la muerte & 0,211 & 0,416 & 1,58 & 0,455 \\
Aceptación neutral & 0,217 & 0,414 & 2,29 & 0,318 \\
Actitud general & 2,177 & 0,015 & 10,40 & 0,006 \\
\hline
\end{tabular}




\section{Resultados}

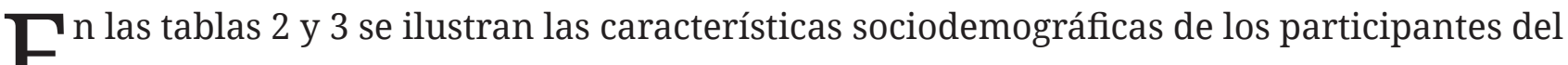
Eestudio, donde se describe, entre otras cosas, información relacionada con el ejercicio profesional. Estos datos fueron obtenidos a través de una encuesta que cada participante diligenció junto con el PAM-R. Para el caso del nivel socioeconómico, se tuvo en cuenta el estrato de la vivienda de los participantes, que, según el Departamento Administrativo Nacional de Estadística (DANE), se puede clasificar como bajo, medio o alto.

Tabla 2. Características sociodemográficas de la población: variables cuantitativas

\begin{tabular}{ccc}
\hline Características & Promedio $\pm \mathrm{DE}$ & Mediana (Q1-Q3) \\
\hline Tiempo de ejercicio profesional, años & $4,65 \pm 3,0$ & $4(3-5)$ \\
Horas de trabajo semanal & $50,1 \pm 10,67$ & $48(48-48)$ \\
Edad & $28,59 \pm 3,48$ & $29(26-30)$ \\
\hline
\end{tabular}

Se destaca que el promedio de tiempo de ejercicio profesional fue 4,65 \pm DE 3,0 y la mayoría pertenecía a la uci plena (76,47\%).

Tabla 3. Características sociodemográficas de la población: variables cualitativas

\begin{tabular}{lcc}
\hline & Características & $\mathbf{n}(\mathbf{\%})$ \\
\hline Tipo de ucI & UcI intermedia & $4(23,53)$ \\
& UcI plena & $13(76,47)$ \\
\hline Sexo & Mujer & \\
& Hombre & $9(52,94)$ \\
\hline Nivel socioeconómico & & $8(47,06)$ \\
& Bajo & \\
& Medio & $2(11,76)$ \\
\hline Estado civil & & $15(88,24)$ \\
& Soltero & $9(52,94)$ \\
& Casado & $4(23,53)$ \\
& Unión libre & $4(23,53)$ \\
\hline
\end{tabular}


La tabla 4 muestra los promedios y las medianas de cada tipo de actitud frente a la muerte. El promedio general de la escala de actitudes fue de 133,53 \pm 21,50. La actitud con mayor promedio fue la de aceptación de acercamiento, seguida por la actitud de aceptación neutral y miedo a la muerte.

Tabla 4. Actitudes hacia la muerte en el personal de la UCI

\begin{tabular}{lcc}
\hline \multicolumn{1}{c}{ Dimensión } & Promedio $\pm \mathrm{DE}$ & Mediana (Q1-Q3) \\
\hline Actitud general & $133,53 \pm 21,50$ & $141(124-148)$ \\
Aceptación de acercamiento & $48,17 \pm 12,64$ & $47(45-55)$ \\
Evitación de la muerte & $17,23 \pm 7,46$ & $17(10-24)$ \\
Aceptación de escape & $16,26 \pm 6,93$ & $19(9-21)$ \\
Miedo a la muerte & $23,0 \pm 7,42$ & $20(17-28)$ \\
Aceptación neutral & $28,94 \pm 4,62$ & $29(28-32)$ \\
\hline
\end{tabular}

Las respuestas agrupadas según la actitud identificada se exponen en la tabla 5. En esta se pueden observar preguntas relacionadas con las creencias sobre la propia muerte de acuerdo con las creencias religiosas, espirituales, entre otras.

Tabla 5. Respuestas de mayor frecuencia a cada una de las preguntas agrupadas por dimensiones de la escala PAM-R

\begin{tabular}{llrr}
\hline \multicolumn{1}{c}{ Características } & n (\%) & $\begin{array}{c}\text { Categoría de la escala } \\
\text { de Likert de mayor } \\
\text { frecuencia }\end{array}$ \\
\hline \multicolumn{1}{c}{ Aceptación de acercamiento } \\
\hline 4. $\quad$ Creo que iré al cielo cuando muera. & $6(35,3)$ & Totalmente de acuerdo \\
8. $\quad$ La muerte es la entrada a un lugar de satisfacción definitiva. & $5(29,4)$ & Bastante de acuerdo \\
13. Creo que el cielo será un lugar mucho mejor que este mundo. & $4(23,5)$ & Bastante de acuerdo \\
15. La muerte es la unión con Dios y con la gloria eterna. & $6(35,3)$ & Totalmente de acuerdo \\
16. La muerte trae la promesa de una vida nueva y gloriosa. & $6(35,3)$ & Bastante de acuerdo \\
22. Ansío reunirme con mis seres queridos después de morir. & $3(17,7)$ & Totalmente de acuerdo \\
25. Veo la muerte como un pasaje a un lugar eterno y bendito. & $4(23,5)$ & Algo de acuerdo \\
27. La muerte ofrece una maravillosa liberación al alma. & $7(41,2)$ & Indeciso \\
28. Una cosa que me consuela al afrontar la muerte es mi creencia en una & $7(41,2)$ & Totalmente de acuerdo \\
vida después de esta. & & \\
31. Espero una vida después de la muerte. & $5(29,4)$ & Indeciso \\
\hline
\end{tabular}




\section{Evitación de la muerte}

3. Evito a toda costa los pensamientos sobre la muerte.

10. Cuando el pensamiento de la muerte entra en mi mente, intento apartarlo.

12. Siempre intento no pensar en la muerte.

19. Evito totalmente pensar en la muerte.

26. Intento no tener nada que ver con el tema de la muerte.

\section{Aceptación de escape}

5. La muerte pondrá fin a todas mis preocupaciones.

$6(35,3) \quad$ Totalmente desacuerdo

9. La muerte proporciona un escape de este mundo terrible.

11. La muerte es una liberación del dolor y el sufrimiento.

23. Veo la muerte como un alivio del sufrimiento terrenal.

29. Veo la muerte como un alivio de la carga de esta vida.

\section{Miedo a la muerte}

1. La muerte es sin duda una experiencia horrible.

$4(23,5) \quad$ Bastante en desacuerdo

2. La perspectiva de mi propia muerte despierta mi ansiedad.

$5(29,4) \quad$ Totalmente de acuerdo

7. Me trastorna la finalidad de la muerte.

$5(29,4) \quad$ Totalmente desacuerdo

18. Tengo un miedo intenso a la muerte.

$6(35,3)$ Bastante en desacuerdo

20. El tema de una vida después de la muerte me preocupa mucho.

$7(41,2) \quad$ Totalmente desacuerdo

21. Me asusta el hecho de que la muerte signifique el fin de todo tal como lo conozco.

32. Me preocupa la incertidumbre de no saber qué ocurre después de la muerte.

\section{Aceptación neutral}

6. La muerte se debería ver como un acontecimiento natural, innegable $11(64,7)$ Totalmente de acuerdo e inevitable.

14. La muerte es un aspecto natural de la vida.

$12(70,6)$ Totalmente de acuerdo

17. No temo a la muerte, pero tampoco deseo que llegue.

$7(41,2) \quad$ Totalmente de acuerdo

24. La muerte es simplemente una parte del proceso de la vida.

$9(52,9) \quad$ Totalmente de acuerdo

30. La muerte no es ni buena ni mala.

En el análisis realizado de las variables sociodemográficas con las respuestas al cuestionario, se logró identificar que existe una correlación moderada y directa entre el promedio general de la escala de actitudes hacia la muerte y los años de trabajo en el servicio, rho $=0,59$, $p=0,016$, tal y como se muestra en la figura 1 . 


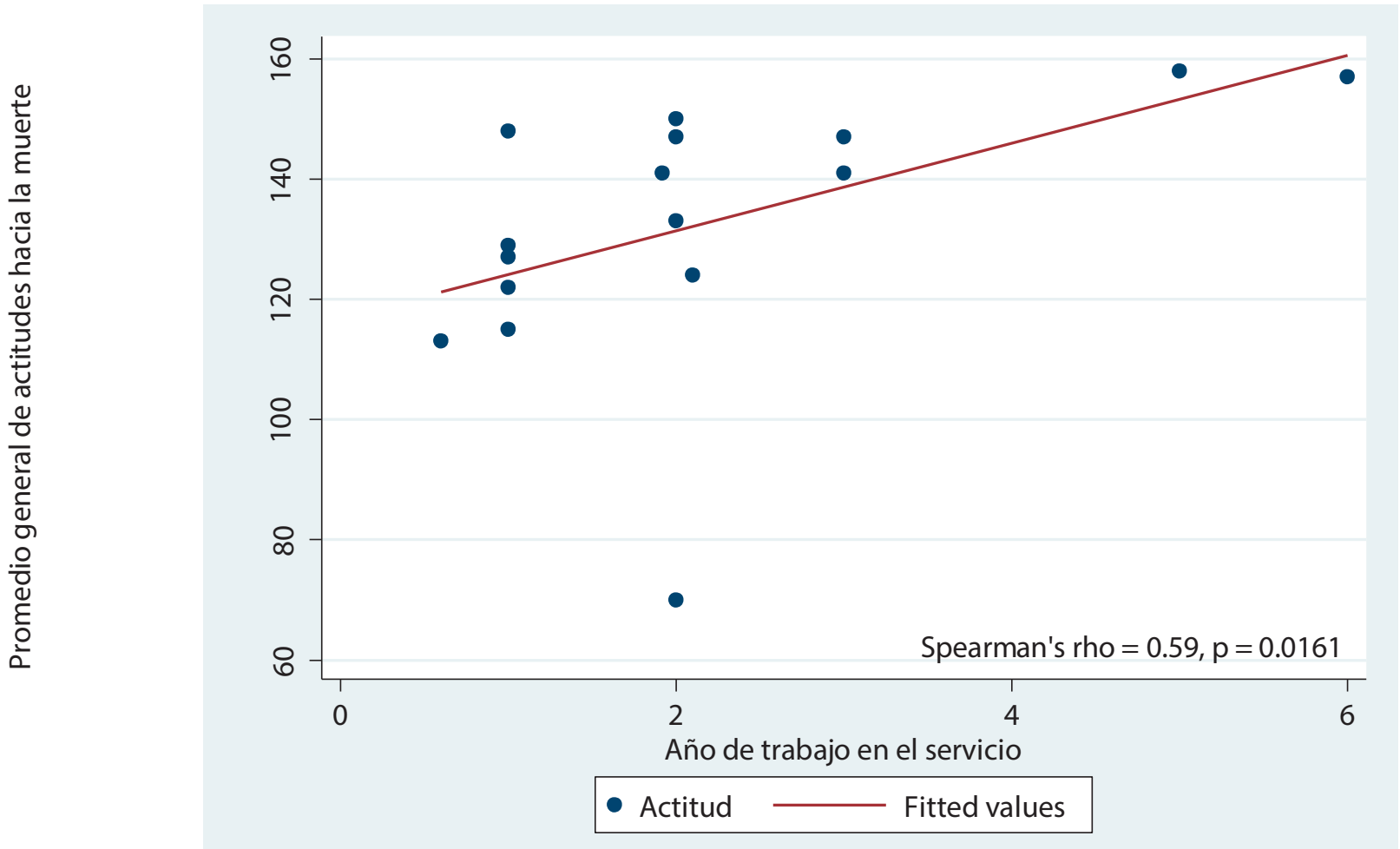

Figura 1. Correlación entre el promedio general de la escala PAM-R y los años de trabajo en la unidad de los participantes (elaborada en Stata, versión 14.0)

\section{Discusión}

$\mathbf{E}^{\mathrm{l}}$ presente artículo describe las actitudes hacia la muerte de un grupo de profesionales Ede enfermería en una uci, a través del instrumento pam-R, elaborado por Gesser, Wong y Reker (9). Como resultado más importante se encontró que, de las cinco dimensiones evaluadas por el instrumento en mención, la actitud predominante fue la de aceptación de acercamiento, la cual es una actitud que se relaciona con creencias religiosas, en donde se tiene una mayor confianza y está presente la creencia de una vida feliz después de la muerte.

Los resultados del presente estudio son en cierta medida similares a lo descrito por García, quien realizó un análisis con estudiantes de primer semestre de medicina, hallando que las mujeres tenían un promedio mayor en la dimensión de evitación de la muerte en contraste con los hombres, quienes tuvieron un promedio mayor en la actitud de aceptación de acercamiento, encontrándose diferencias estadísticamente significativas ( $p>0,028)(12)$. En este trabajo, no se reportaron diferencias significativas entre géneros, pero sí se descubrió una correlación moderada y directa entre el promedio general de la escala de actitudes hacia la muerte y los años de trabajo en el servicio, resaltando que la diferencia entre las poblaciones 
evaluadas fue el grado de formación académica. No se evidencia en la literatura consultada una explicación clara a este hallazgo, sin embargo, podría deberse a la experiencia adquirida en el manejo de situaciones críticas, incluyendo el quehacer frente a la muerte.

Algunos estudios realizados con profesionales de enfermería, como el de Fernández y colaboradores en 2017, quienes evaluaron las actitudes ante la muerte de enfermeras que laboraban en una unidad de oncología, hematología y cuidados paliativos, no lograron evidenciar una relevancia notable entre las diferentes actitudes, según la escala PAM-R (13). Esto sugiere la necesidad de tener en cuenta variables como edad, condiciones familiares, formación académica y años de experiencia, que puedan explicar estas diferencias al evaluar actitudes hacia la muerte, algo que ha sido descrito recientemente por Ay y colaboradores, quienes reportaron diferencias significativas en las actitudes de enfermeras en Turquía de acuerdo con su estado civil, el tener hijos y los años de experiencia (14). En este sentido, nuestro estudio no mostró diferencias significativas en ninguna de estas variables.

En cuanto al desarrollo de este tema en el nororiente colombiano, se llevó a cabo un estudio en la ciudad de Bucaramanga por Álvarez-Ramírez, donde se describieron actitudes de miedo y aceptación de acercamiento hacia la muerte en adolescentes y adultos tempranos (8). Estos resultados guardan relación con respecto a la edad de los participantes, ya que la edad promedio fue de 28 años, muy similar a la de los adultos incluidos en el presente estudio; no obstante, no se encontraron estudios que evalúen las actitudes del personal de salud en nuestra región.

Con respecto a la actitud de aceptación neutral, se ha descrito que es propia de adultos mayores, como es evidenciado en la tesis de Morales Pérez, en donde la actitud neutral fue la más predominante del grupo evaluado $(45 \%, \mathrm{n}=100)$, datos similares a los encontrados por Uribe y Valderrama, y al estudio de Velásquez en el año 2015 con adultos mayores institucionalizados $(9,15,16)$. Estos mismos hallazgos han sido descritos en nuestro país por autores como Ojeda y colaboradores (17). Sin embargo, la aceptación neutral fue la segunda actitud más predominante en los profesionales de salud del presente estudio, siendo una gran diferencia la edad, lo que podría sugerir que la actitud es independiente de esta variable. En este sentido, en un reciente estudio con 376 adultos jóvenes de nacionalidad española, se halló el mayor puntaje para la aceptación neutral, lo que reafirma la necesidad de revisar otras variables sociodemográficas como profesión, género y edad (18). Estos hallazgos de neutralidad independiente de la edad han sido descritos por Uribe Rodríguez y colaboradores, quienes compararon la actitud de adultos mayores versus la actitud de adultos jóvenes, encontrando la neutralidad como predominante en ambos grupos. Estos autores deducen que pudiera deberse a que el tema de la muerte ya no es algo discutido solo por los adultos mayores, y por lo tanto, existe una mayor cercanía y percepción de la muerte en ambos grupos etarios (19).

Además de la actitud de aceptación neutral y como se comentó anteriormente, el pAM-R evalúa una serie de actitudes como el miedo a la muerte, la evitación a la muerte, la aceptación y escape, y la aceptación de acercamiento. Esta última fue la actitud con mayor porcentaje en 
el presente estudio. Según los autores de este instrumento, esta actitud se asocia con creencias religiosas conforme con las cuales se tiene mayor confianza en una vida feliz después de la muerte (9). En este sentido, se ha sugerido la importancia de comprender los valores y creencias ante la muerte y del proceso de morir, así como también las actitudes y acciones relacionadas con las cuestiones de lo cotidiano que influyen en la vida personal y profesional, tal como lo describieron Souza y colaboradores en su artículo sobre sentimientos de los enfermeros en relación con la muerte y el proceso de morir, en el que se evidencia que si bien los entrevistados dijeron ver a la muerte como un hecho natural, se logró identificar mecanismos de defensa ante el dolor y el sufrimiento (20). Que esta actitud esté seguida de la neutralidad podría deberse a la falta de experiencia o formación con respecto a la muerte, debido a que, en general, el entrenamiento para el profesional de enfermería que labora en uci incluye una serie de temas clínicos y operativos, dejando de lado procesos como el duelo y la muerte.

Finalmente, y como recomendación, efectuar una inmersión en el tema de enfermería y el final de la vida desde la formación académica de pregrado pudiera dar nuevas luces para entender las actitudes frente a la muerte, como ya se ha sugerido en otros países $(21,22)$. Adicionalmente, deben seguirse haciendo investigaciones de tipo cualitativo y cuantitativo que permitan comprender las actitudes de la enfermera como profesional y como persona, y cómo esto influye en la relación con su sujeto de cuidado. Otra recomendación para futuras investigaciones tiene que ver con un mayor tamaño de la muestra, el cual fue una limitación del presente estudio. De igual manera, involucrar enfermeras de diferentes unidades asistenciales redundaría en un conocimiento mayor del tema.

En conclusión, dentro de las actitudes hacia la muerte evaluadas en las enfermeras de ucI que participaron en el presente estudio, la que más predominó fue la de aceptación de acercamiento, seguida de la aceptación neutral. En este sentido, se sugiere que la educación en temas relacionados con el final de la vida podría lograr cambios en estas actitudes, las cuales son dinámicas, como lo describieron recientemente Hong y colaboradores, quienes afirman que este tipo de entrenamiento genera actitudes proactivas y positivas sobre la muerte (23).

Los resultados de este estudio refieren la necesidad de continuar un proceso de investigación e intervención con enfermeras de uci, dado que, por ser cuidadoras directas y estar en constante contacto con la muerte, sus actitudes pueden influenciar en ellas mismas y en sus sujetos de cuidado.

\section{Conflicto de intereses}

Ninguno declarado. 


\section{Descargos de responsabilidad}

Droducto derivado del proyecto: "Cuantificación del tiempo de las actividades de enfer1 mería en una unidad de cuidados intensivos”. Estudio: Caretime. Financiado por Conadi Consejo Nacional de Investigaciones (Conadi ucc, código 2244).

\section{Referencias}

1. Carmona-Monge FJ, Uria K, García S, Quirós C, Bergaretxe M, Etxabe G, et al. Análisis de uso del puntaje de las actividades de enfermería en dos icus españoles. Rev Esc Enferm UsP. 2013;47(5):1108-16. Doi: 10.1590/S0080-623420130000500014

2. Dennis RJ, Pérez A, Rowan K, Londoño D, Metcalfe A, Gómez C, et al. Factores asociados con la mortalidad hospitalaria en pacientes admitidos en cuidados intensivos en Colombia. Arch Bronconeumol. 2002;38(3):117-22.

3. Portilla Reyes LA, Rojas Rengifo B, Niño de Guzmán Riveros MY. Actitud del profesional de enfermería frente a la muerte en la unidad de cuidados intensivos en un hospital nacional, 2018 [trabajo académico]. [Lima]: Universidad Peruana Cayetano Heredia; 2018. Disponible en: http://repositorio.upch.edu.pe/handle/upch/3521

4. Zambrano Plata G. Estresores en las unidades de cuidado intensivo. Aquichan. 2009;6(1).

5. Díaz M, Juarros N, García B, Sáez C. Study on anxiety in intensive care nursing professionals facing the process of death. Burgos University Hospital (HUBU); 2017. Doi: 10.6018/ eglobal.16.1.232221

6. Alonso Salido L. Ansiedad del personal de enfermería en relación a la muerte de sus pacientes. Comparativa uci y oncología [trabajo de grado]. [Leioa]: Universidad del País Vasco; 2014.

7. Acosta A, España C, Jiménez K, Suárez J, Ortiz S, Vanegas Y. Capacidad de afrontamiento de las enfermeras ante la muerte en las unidades de cuidados intensivos pediátrica y neonatales en Cartagena, 2016. Cartagena de Indias: Universidad de Cartagena; 2016.

8. Álvarez-Ramírez LY. Actitudes frente a la muerte en un grupo de adolescentes y adultos tempranos en la ciudad de Bucaramanga. Aquichan. 2009;9(2):156-70.

9. Uribe Rodríguez AF, Valderrama Orbegozo L, López S. Actitud y miedo ante la muerte en adultos mayores. Pensamiento Psicológico. 2007;3(8):109-20.

10. Colombia, Ministerio de Salud. Resolución 8430 (1993 oct 4).

11. Asociación Médica Mundial. Declaración de Helsinki sobre principios éticos para las investigaciones médicas en seres humanos, adoptada por la 59a Asamblea General. Seúl; 2008.

12. Mercado GLR, García RA, Arceo GME, Pimentel RML, Díaz FM, Arauz CJ. Actitud hacia la muerte y su relación con la empatía médica en estudiantes de Medicina. Revista Cubana de Educación Médica Superior. 2016;30(1). 
13. Fernández Fernández JA, García Martínez L, García Bueno ML, Fernández Grande AN, Lozano González T, Rubio Prieto A. Actitudes y afrontamiento ante la muerte en el personal de enfermería. Tiempos de Enfermería y Salud = Nursing and Health Times. 2017;2: 27-33.

14. Ay MA, Öz F. Nurses attitudes towards death, dying patients and euthanasia: a descriptive study. Nursing Ethics. 2019;26(5):1442-57. Doi: 10.1177/0969733017748481

15. Morales Pérez KR. Actitud ante la muerte e intensidad de duelo en adultos mayores con pérdida del cónyuge adscritos a la unidad de medicina familiar número 64 del Instituto Mexicano del Seguro Social en el período comprendido de marzo-agosto de 2013 [tesis]. [Toluca]: Universidad Autónoma del Estado de México; 2014.

16. Velásquez Crespo RE. Actitud hacia la muerte en pacientes geriátricos institucionalizados [tesis de grado]. [La Antigua Guatemala]: Universidad Rafael Landívar; 2015.

17. Ojeda España TB. Actitud ante la muerte: un estudio comparativo con adultos mayores según género. La Antigua Guatemala, Sacatepéquez [tesis]. [Guatemala]: umG; 2016.

18. Clemente Gutiérrez T. Ansiedad, miedo y actitudes hacia la muerte en población general mayor de edad: evaluación y repercusión en variables psicológicas y de salud. Universidad de Salamanca; 2018. Disponible en: http://hdl.handle.net/10366/137927

19. Uribe Rodríguez AF, Valderrama L, Durán Vallejo DM, Galeano Monroy C, Gamboa K, López S. Diferencias evolutivas en la actitud ante la muerte entre adultos jóvenes y adultos mayores. Acta Colombiana de Psicología. 2008;11(1):119-26.

20. Souza e Souza LP, Mota Ribeiro J, Barbosa RR, Ribeiro Gonçalves RC, Oliveira e Silva CS, Barbosa DA. La muerte y el proceso de morir: sentimientos manifestados por los enfermeros. Enferm Glob. 2013; 12(32):222-9.

21. Lin J, Supiano KP, Madden C, McLeskey N. The impact of the end-of-life nurse education consortium on attitudes of undergraduate nursing students toward care of dying patients. J Hosp Palliat Nurs. 2018;20(4):340-8. Doi: 10.1097/NJH.0000000000000445

22. Chen CH, Huang LC, Liu HL, Lee HY, Wu SY, Chang YC, et al. To explore the neonatal nurses' beliefs and attitudes towards caring for dying neonates in Taiwan. Matern Child Health J. 2013;17(10):1793-801. Doi: 10.1007/s10995-012-1199-0

23. Hong M, Hong S, Adamek ME, Kim MH. Death attitudes among middle-aged Koreans: role of end-of-life care planning and death experiences. Int J Aging Hum Dev. 2018;86(1):5168. Doi: $10.1177 / 0091415016689473$ 